\title{
Related oscillation criteria for higher order self-adjoint differential and integro-differential equations\$
}

\author{
William T. Reid $\dagger$ \\ The University of Texas at Austin, Austin, Texas, U.S.A. \\ (Communicated by Professor W.N. Everitt) \\ (MS received 8 July 1977. Read 24 April 1978.)
}

\section{INTRODUCTION}

Within recent years considerable attention has been devoted to extensions of the classical Sturmian theory of real linear homogeneous differential equations of the second order. In particular, such extensions have included not only self-adjoint systems of differential equations, but also higher order self-adjoint differential and integro-differential equations. For problems in these latter categories, however, only limited attention has been given to detailed application of the general oscillation and comparison criteria. The present paper is devoted to this area, and, in particular, it is shown how existing criteria may be exploited to obtain comparison theorems between equations of different orders. Although the presented results have ready extensions to vector differential and integrodifferential equations of higher order, [see, for example, 5, 6, 7], for simplicity attention is restricted to scalar equations. Section 2 is devoted to the statement of known general criteria of oscillation for self-adjoint equations of higher order, with special applications of these criteria presented in Section 3. Finally, Section 4 sketches the framework of corresponding applications for self-adjoint higher order integro-differential equations.

For $I$ an interval on the real line $R$, the symbols $\mathfrak{E}(I)$ and $\overleftarrow{\complement}^{m}(I)$ will denote the class of functions $f: I \rightarrow R$ which are respectively continuous, and continuously differentiable of the first $m$ orders. If $[a, b]$ is a non-degenerate compact subinterval of $I$ then $\mathfrak{L}[a, b], \mathfrak{R}^{\infty}[a, b], \mathfrak{R}^{2}[a, b], \mathfrak{A}[a, b], \mathfrak{A}^{m}[a, b], \mathfrak{A}^{m \cdot 2}[a, b]$ will designate respectively the classes of functions $f:[a, b] \rightarrow R$ which are (Lebesgue) integrable, (Lebesgue) measurable and essentially bounded, measurable with $f^{2}$ integrable, a.c. (absolutely continuous), of class ${ }^{(5-1}[a, b]$ with $f^{(m-1)} \in$ $\mathfrak{A}[a, b]$, of class $\mathfrak{Q}^{m}[a, b]$ and $f^{(m)} \in \mathfrak{R}^{2}[a, b]$.

\section{Basic Results on Oscillation}

For $j=0,1, \ldots, n$ let $p_{j}: I \rightarrow R$ be real-valued functions defined on an interval $I$ of the real line, and satisfying the following hypothesis.

\$Presented as the second lecture in the series of John Barrett Memorial Lectures. The University of Tennessee, Knoxville, Tennessee, May 1977.

$\dagger$ The Society regrets to record that the author died in October 1977. 
$\left(\mathfrak{Q}_{n}\right) p_{n}(t) \neq 0$ for $t \in I$, and $p_{n}, 1 / p_{n}, p_{\alpha},(\alpha=0,1, \ldots, n-1)$, belong to $\mathfrak{L}^{\infty}[a, b]$ for arbitrary non-degenerate compact subintervals $[a, b]$ of $I$.

If $[a, b]$ is a non-degenerate compact subinterval of $I$ the linear vector space of real-valued functions $y \in \mathfrak{A}^{n, 2}[a, b]$ will be denoted by $\mathscr{D}^{n}[a, b]$, and $\mathscr{D}_{0}^{n}[a, b]$ will designate the subspace of elements $y \in \mathscr{D}^{n}[a, b]$ satisfying $y^{(\alpha)}(a)^{*} 0=$ $y^{(\alpha)}(b),(\alpha=0,1, \ldots, n-1)$. For $y$ and $z$ elements of $\mathscr{D}^{n}[a, b]$ the integral

$$
\mathbf{J}^{n}[y, z \mid a, b]=\int_{a}^{b}\left\{\sum_{j=0}^{n} p_{j}(t) z^{(j)}(t) y^{(j)}(t)\right\} d t
$$

is well-defined, and is a symmetric form on $\mathscr{D}^{n}[a, b] \times \mathscr{D}^{n}[a, b]$; as usual, the notation $\mathrm{J}^{n}[y, y \mid a, b]$ is contracted to $\mathrm{J}^{n}[y \mid a, b]$.

If $u \in \mathscr{D}^{n}[a, b]$, then the condition

$$
\mathbf{J}^{n}[u, z \mid a, b]=0 \text { for } z \in \mathscr{D}_{0}^{n}[a, b]
$$

holds iff $u$ is a solution on $[a, b]$ of the quasi-differential equation

$$
L_{2 n}[u](t)=p_{0}(t) u(t)-\left\{p_{1}(t) u^{\prime}(t)-\left\{\cdots-\left\{p_{n}(t) u^{(n)}(t)\right\}^{\prime} \cdots\right\}^{\prime}\right\}^{\prime}=0 .
$$

In conformity with usual terminology, a function $u$ is called a solution of (2.3) on $[a, b]$ if $u \in \mathscr{D}^{n}[a, b]$ and there exist corresponding functions $v_{k}(t)=v_{k}(t \mid u)$ of class $\mathfrak{A}[a, b]$ satisfying a.e. (almost everywhere) on $[a, b]$ the recursive relations

$$
\begin{aligned}
& v_{n}(t)=p_{n}(t) u^{(n)}(t), \\
& v_{n-r}(t)=p_{n-r}(t) u^{(n-r)}(t)-v_{n-r+1}^{\prime}(t), \quad(r=1, \ldots, n-1),
\end{aligned}
$$

and

$$
L_{2 n}[u](t) \equiv p_{0}(t) u(t)-v_{1}^{\prime}(t)=0 .
$$

The above statements may be established by the classical proof of the fundamental lemma of the calculus of variations, [see, for example, 5; pp. 797 798], and in essence states that (2.3) or (2.5) is the Euler-Lagrange differential equation for the functional $\mathrm{J}^{n}[y \mid a, b]$ on the class of admissible functions $\mathscr{D}^{n}[a, b]$. Alternately, the pair of $n$-dimensional vector functions $\mathbf{u}(t)=\left(u^{(k-1)}(t)\right)$, $\mathbf{v}(t)=\left(v_{k}(t \mid u)\right),(k=1, \ldots, n)$, are solutions in the Carathéodory sense of the associated first order Hamiltonian system of differential equations, [6; Sec. 2]. In particular, if $p_{j} \in \Im^{j}(I),(j=0,1, \ldots, n)$, a function $u$ is a solution of (2.3) iff $u \in \mathscr{D}^{n}[a, b]$ and $p_{k} u^{(k)} \in \mathfrak{A}^{k}[a, b],(k=1, \ldots, n)$, for arbitrary compact subintervals $[a, b]$ of $I$, and for $t$ a.e. on $I$,

$$
L_{2 n}[u](t) \equiv \sum_{j=0}^{n}(-1)^{j}\left[p_{j}(t) u^{(j)}(t)\right]^{(j)}=0 .
$$

Two distinct values $t_{1}, t_{2}$ on $I$ are called (mutually) conjugate with respect to (2.3) if there exists a non-identically vanishing solution $u(t)$ of this equation satisfying $u^{(\alpha)}\left(t_{1}\right)=0=u^{(\alpha)}\left(t_{2}\right),(\alpha=0,1, \ldots, n-1)$. If $I_{0}$ is a non-degenerate subinterval of $I$ such that no two distinct points of $I_{0}$ are conjugate, then this quasi-differential equation is said to be disconjugate on $I_{0}$. Also, if for distinct $t_{1}, t_{2}$ on $I$ there exists a non-identically vanishing solution $u(t)$ of $(2.3)$ such that $v_{k}\left(t_{1} \mid u\right)=0, u^{(k-1)}\left(t_{2}\right)=0,(k=1, \ldots, n)$, then $t_{2}$ is said to be focal to $t_{1}$. 
If $u_{1}$ and $u_{2}$ are solutions of $(2.3)$ and $v_{\sigma}(t)=\left(v_{k \sigma}(t)\right)=\left(v_{k}\left(t \mid u_{\sigma}\right)\right),(\sigma=1$, 2), are the corresponding functions (2.4), then $\left\{u_{1} \mid u_{2}\right\}(t) \equiv$ $\sum_{k=1}^{n}\left[v_{k 2}(t) u_{1}^{(k-1)}(t)-u_{2}^{(k-1)}(t) v_{k 1}(t)\right]$ is constant on I. If $\left\{u_{1} \mid u_{2}\right\} \equiv 0$ then the solutions $u_{1}, u_{2}$ are said to be (mutually) conjugate. A set of $q$ linearly independent solutions of (2.3) which are mutually conjugate form a basis for a conjugate family of solutions of dimension $q$, consisting of the linear combinations of these solutions. For brevity, the solutions of a conjugate family of dimension $n$ will be referred to as a conjugate basis for (2.3). In particular, if for $s \in I$ the solutions $u_{\beta}^{0}(t)=u_{\beta}^{0}(t ; s)$ and $u_{\beta}^{1}(t)=u_{\beta}^{1}(t ; s),(\beta=1, \ldots, n)$, are determined by the initial conditions

(i) $u_{\beta}^{0(\alpha-1)}(s ; s)=0, \quad v_{\alpha \beta}^{0}(s ; s)=v_{\alpha}\left(s \mid u_{\beta}^{0}\right)=\delta_{\alpha \beta}$,

(ii) $u_{\beta}^{1(\alpha-1)}(s ; s)=\delta_{\alpha \beta}, \quad v_{\alpha \beta}^{1}(s ; s)=v_{\alpha}\left(s \mid u_{\beta}^{1}\right)=0$,

then each of the families $\left\{u_{\beta}^{0}(t ; s)\right\}$ and $\left\{u_{\beta}^{1}(t ; s)\right\}$ is a conjugate basis for (2.3). Moreover, a value $s_{1} \neq s$ of $I$ is conjugate to $s$, or focal to $s$, in each case of index $r$, according as the $n \times n$ matrix $\left[u_{\beta}^{\alpha(\alpha-1)}\left(s_{1} ; s\right)\right]$ or $\left[u_{\beta}^{1(\alpha-1)}\left(s_{1} ; s\right)\right]$ is of rank $n-r$.

For $[a, b]$ a non-degenerate compact subinterval of $I$ we set $\mathscr{D}_{* 0}^{n}[a, b]=$ $\left\{y: y \in \mathscr{D}^{n}[a, b], y^{(\alpha)}(b)=0, \alpha=0,1, \ldots, n-1\right\}$ and $\mathscr{D}_{0 *}^{n}[a, b]=\left\{y: y \in \mathscr{D}^{n}[a, b]\right.$, $\left.y^{(\alpha)}(a)=0, \alpha=0,1, \ldots, n-1\right\}$. Also, for a uniformity of notation in some of the subsequent discussion we alternatively use $\mathscr{D}_{00}^{n}[a, b]$ for the class designated earlier by $\mathscr{D}_{0}^{n}[a, b]$; clearly, $\mathscr{D}_{\mathrm{o} 0}^{n}[a, b]=\mathscr{D}_{* 0}^{n}[a, b] \cap \mathscr{D}_{0 *}^{n}[a, b]$.

Basic results for the consideration of oscillation phenomena for equations of the form (2.3) are presented in the following theorem; for a detailed derivation of these results, see $[4 ; \mathrm{Ch}$. VII].

THEOREM 2.1. Suppose that hypothesis $\left(\mathfrak{S}_{n}\right)$ is satisfied, and $[a, b]$ is a non-degenerate subinterval of $I$. Then $\mathrm{J}^{n}[y \mid a, b]$ is positive definite on $\mathscr{D}_{0}^{n}[a, b]$ iff $p_{n}(t) \geq 0$ for $t$ a.e. on $[a, b]$, and one of the following conditions holds:

(i) (2.3) is disconjugate on $[a, b]$;

(ii) the conjugate basis $\left\{u_{\beta}^{0}(t ; a)\right\}$ determined as in (2.6i) is such that the $n \times n$ Wronskian $\left[u_{\beta}^{0(\alpha-1)}(t ; a)\right]$ is non-singular on $(a, b]$;

(iii) there exists a conjugate basis $\left\{u_{\beta}(t)\right\}$ of (2.3) such that the $n \times n$ Wronskian $\left[u_{\beta}^{(\alpha-1)}(t)\right]$ is non-singular on $[a, b]$.

Correspondingly, $\mathbf{J}^{n}[y \mid a, b]$ is positive definite on $\mathscr{D}_{* 0}^{n}[a, b]$ iff $p_{n}(t) \geq 0$ for $t$ a.e. on $[a, b]$, and one of the following conditions holds:

(i') there is no point on $(a, b]$ focal to $t=a$;

(ii') the conjugate basis $\left\{u_{\beta}^{1}(t ; a)\right\}$ determined as in (2.6ii) is such that the $n \times n$ Wronskian $\left[u_{\beta}^{1(\alpha-1)}(t ; a)\right]$ is non-singular on $[a, b]$.

In the following, $\left(\mathfrak{B}_{n}^{+}\right)$will designate the following hypothesis.

$\left(\mathfrak{Q}_{n}^{+}\right)$Hypothesis $\left(\mathfrak{G}_{n}\right)$ is satisfied, and $p_{n}(t)>0$ for $t \in I$.

Other criteria for the existence of conjugate and focal points involve the eigenvalues of boundary problems associated with the differential operator $L_{2 n}[u]$. For $[a, b] \subset I$, let $B_{00}\left[a, b \mid L_{2 n}\right], B_{* 0}\left[a, b \mid L_{2 n}\right], B_{0 *}\left[a, b \mid L_{2 n}\right]$, denote the 
two-point boundary problem involving

$$
L_{2 n}[u](t)-\lambda u(t)=0,
$$

and the respective boundary conditions:

$$
\begin{array}{lll}
\Delta_{00}[a, b]: u^{(\alpha-1)}(a)=0, & u^{(\alpha-1)}(b)=0, & (\alpha=1, \ldots, n) ; \\
\Delta_{* 0}[a, b]: v_{\alpha}(a \mid u)=0, & u^{(\alpha-1)}(b)=0, & (\alpha=1, \ldots, n) ; \\
\Delta_{0 *}[a, b]: u^{(\alpha-1)}(a)=0, & v_{\alpha}(b \mid u)=0, & (\alpha=1, \ldots, n) .
\end{array}
$$

THEOREM 2.2. If hypothesis $\left(\mathfrak{S}_{n}^{+}\right)$holds, then for $(p, q)$ either $(0,0),(*, 0)$ or $\left(0,{ }^{*}\right)$ the boundary problem $B_{p q}\left[a, b \mid L_{2 n}\right]$ possesses a sequence of eigenvalues $\lambda_{j}^{p q}=\lambda_{j}^{p q}[a, b]$ satisfying $\lambda_{1}^{p q} \leq \lambda_{2}^{p q} \leq \cdots$, and corresponding eigenfunctions $u=$ $u_{j}^{p q}(t)=u_{j}^{p q}(t \mid a, b)$ of $(2.7)$ for $\lambda=\lambda_{j}^{p q}$ such that

(a) $\int_{a}^{b} u_{j}^{p q}(t) u_{k}^{p q}(t) d t=\delta_{j k}, \quad(j, k=1,2, \ldots)$;

(b) $\lambda_{1}^{p q}=\mathbf{J}^{n}\left[u_{1}^{p q} \mid a, b\right]$ is the minimum of $\mathbf{J}^{n}[y \mid a, b]$ on $\left\{y: y \in \mathscr{D}_{p q}[a, b]\right.$, $\left.\int_{a}^{b} y^{2}(t) d t=1\right\}$

(c) for $j=2,3, \ldots$ the class $\left\{y: y \in \mathscr{D}_{p q}[a, b], \int_{a}^{b} y^{2}(t) d t=1, \int_{a}^{b} u_{i}^{p q}(t) y(t) d t=\right.$ $0, i=1, \ldots, j-1\}$ is non-empty and $\lambda_{j}^{p a}$ is the minimum of $\mathrm{J}^{n}[y \mid a, b]$ on this class;

(d) $\lambda_{j}^{p a}[a, b] \rightarrow \infty$ as $j \rightarrow \infty$;

(e) As functions of $b$ on $I_{a}^{+}=\{t: t \in I, t>a\}$ the eigenvalues $\lambda_{j}^{00}[a, b]$ and $\lambda_{j}^{* 0}[a, b]$ are continuous strictly monotone decreasing functions and $\lambda_{1}^{00}[a, b] \rightarrow$ $\infty, \quad \lambda^{* 0}[a, b] \rightarrow \infty$ as $b \rightarrow a^{+}$. Correspondingly, as functions of $a$ on $I_{a}^{-}=$ $\{t: t \in I, t<a\}$ the eigenvalues $\lambda_{j}^{00}[a, b]$ and $\lambda_{j}^{0 *}[a, b]$ are continuous strictly monotone increasing functions and $\lambda_{1}^{00}[a, b] \rightarrow \infty, \lambda_{1}^{0 *}[a, b] \rightarrow \infty$ as $a \rightarrow b^{-}$.

For a proof of the results of the above theorem the reader is referred to [4, Ch. VII, Sec. 11 and 6, Sec. 4].

For $s \in I$, let the values of $I_{s}^{+}$that are conjugate to $s$ with respect to (2.3) be ordered as a sequence $\left\{t_{j}^{+}(s)\right\}=\left\{t_{j}^{+}\left(s \mid J^{n}\right)\right\}$, with $t_{1}^{+}(s) \leq t_{2}^{+}(s) \leq \cdots$, and each repeated a number of times equal to its index as a conjugate point; the sequence $\left\{t_{j}^{+}(s)\right\}$ may be infinite, finite, or even vacuous. In view of the results stated in Theorems 2.1 and 2.2, a value $s_{1} \in I_{s}^{+}$is conjugate to $s$ relative to (2.3) iff there exists an integer $m$ such that $\lambda_{m}^{00}\left[s, s_{1}\right]=0$; moreover, the index of $s_{1}$ as conjugate to $s$ is equal to the multiplicity of $\lambda=0$ as an eigenvalue of $B_{00}\left[s, s_{1} \mid L_{2 n}\right]$. Also, the number of values on the open interval $\left(s, s_{1}\right)$ that are conjugate to $s$, with each counted according to its index, is equal to the number of negative eigenvalues of $B_{00}\left[s, s_{1} \mid L_{2 n}\right]$, with each counted according to its multiplicity. Moreover, if $k$ denotes the number of negative eigenvalues of $B_{00}\left[s, s_{1} \mid L_{2 n}\right]$, then $k$ is the dimension of a maximal subspace of $\mathscr{D}_{0}\left[s, s_{1}\right]$ on which $J^{n}\left[y \mid s, b_{1}\right]$ is negative definite. In an alternative form of statement, $k$ is the smallest integer for which there exists a set of $k$ functions $f_{1}, \ldots, f_{k}$ of $\mathscr{D}_{0}^{n}\left[s, s_{1}\right]$ such that if $y \in \mathscr{D}_{0}^{n}\left[s, s_{1}\right]$ and $\int_{s}^{s_{1}} f_{j}(t) y(t) d t=0, j=1, \ldots, k$, then $\mathbf{J}^{n}\left[y \mid s, s_{1}\right] \geq 0$; indeed, one such set of functions $f_{1}, \ldots, f_{k}$ may be chosen as a maximal set of linearly independent solutions of $B_{00}\left[s, s_{1} \mid L_{2 n}\right]$ belonging to negative eigenvalues. In general, this latter condition specifies $k$ as the negative index of $J^{n}$ on 
$\mathscr{D}_{0}^{n}\left[s, s_{1}\right]$, denoted by $n^{-}\left\{\mathbf{J}^{n} / \mathscr{D}_{0}^{n}\left[s, s_{1}\right]\right\}$, a concept which is meaningful without the added condition $p_{n}(t)>0$ of $\left(\mathfrak{S}_{n}^{+}\right)$. For example, if $p_{j}(t) \geq 0,(j=0,1, \ldots, n)$, then $n^{-}\left\{\mathbf{J}^{n} / \mathscr{D}_{0}^{n}[a, b]\right\}=0$. As another example, if $n=2, p_{2}(t) \equiv 0, p_{1}(t) \equiv 1, p_{0}(t) \equiv-1$, and $\pi<b-a<2 \pi$, then $n^{-}\left\{\mathbf{J}^{2} / \mathscr{D}_{0}^{2}[a, b]\right\}=1$. This result may be established by first showing that if $a<a_{1}<b_{1}<b$ and $b_{1}-a_{1}>\pi$ then for $J^{1}\left[y \mid a_{1}, b_{1}\right]=$ $\int_{a_{1}}^{b_{1}}\left\{y^{\prime 2}(t)-y^{2}(t)\right\} d t$ one has $n^{-}\left\{\mathbf{J}^{1} / \mathscr{D}_{0}^{1}\left[a_{1}, b_{1}\right]\right\}=1$, and then using suitable approximation processes to obtain the stated result.

Correspondingly, let the values on $I_{s}^{+}$that are focal to $s$ with respect to (2.3) be ordered as a sequence $\left\{\tau_{j}^{+}(s)\right\}=\left\{\tau_{j}^{+}\left(s \mid J^{n}\right)\right\}$ with $\tau_{1}^{+}(s) \leq \tau_{2}^{+}(s) \leq \cdots$, and each repeated a number of times equal to its index. Then a value $s_{1} \in I_{s}^{+}$is focal to $s$ relative to (2.3) iff there exists an integer $m$ such that $\lambda_{m}^{* 0}\left[s, s_{1}\right]=0$. Moreover, the index of $s_{1}$ as a focal point to $s$ is equal to the multiplicity of $\lambda=0$ as an eigenvalue of $B_{* 0}\left[s, s_{1} \mid L_{2 n}\right]$, and the number of values on the open interval $\left(s, s_{1}\right)$ that are focal to $s$, with each counted according to its index, is equal to the number of negative eigenvalues of $B_{* 0}\left[s, s_{1} \mid L_{2 n}\right]$, with each counted according to its multiplicity. Also, if $k$ denotes the number of negative eigenvalues of $B_{* 0}\left[s, s_{1} \mid L_{2 n}\right]$ counted by multiplicities, then $k$ is the dimension of a maximal subspace of $\mathscr{D}_{* 0}^{n}\left[s, s_{1}\right]$ on which $\mathbf{J}^{n}\left[y \mid s, s_{1}\right]$ is negative definite, there exist $k$ functions $g_{1}, \ldots, g_{k}$ of $\mathscr{D}_{* 0}^{n}\left[s, s_{1}\right]$ such that $\mathbf{J}^{n}\left[y \mid s, s_{1}\right] \geq 0$ for $y \in$ $\left\{y: y \in \mathscr{D}_{* 0}^{n}\left[s, s_{1}\right], \int_{s}^{s_{1}} g_{j}(t) y(t) d t=0, j=1, \ldots, k\right\}$, and corresponding to the notation introduced above for conjugate points we write $n^{-}\left\{\mathbf{J}^{n} / \mathscr{D}_{* 0}^{n}\left[s, s_{1}\right]\right\}=k$.

For values on $I_{s}^{-}=\{t: t \in I, t<s\}$ that are conjugate or focal to $s$, and the associated boundary problems $B_{00}\left[s_{1}, s \mid L_{2 n}\right]$ and $B_{0 *}\left[s_{1}, s \mid L_{2 n}\right], s_{1}<s$, there are results which are dual to those stated above for conjugate and focal points to $s$ on $I_{s}^{+}$, but for brevity they will not be stated explicitly.

In regard to the type of conditions appearing above, and which will be used in subsequent sections, it is to be noted that if $\mathrm{J}^{\mathbf{n}}[y \mid a, b]$ is positive definite on $\mathscr{D}_{0}^{n}[a, b]$ or $\mathscr{D}_{* 0}^{n}[a, b]$, then for $n<N<\infty$ one also has the condition that $\mathbf{J}^{n}[y \mid a, b]$ is positive definite on $\mathscr{D}_{0}^{N}[a, b]$ or $\mathscr{D}_{*_{0}}^{N}[a, b]$, respectively. On the other hand, for $n<N<\infty$ the positive definiteness of $\mathbf{J}^{n}[y \mid a, b]$ on $\mathscr{D}_{0}^{N}[a, b]$ or $\mathscr{D}_{* 0}^{N}[a, b]$ does not imply the positive definiteness of $\mathbf{J}^{n}[y \mid a, b]$ on $\mathscr{D}_{0}^{n}[a, b]$ or $\mathscr{D}^{n}{ }_{00}[a, b]$, but merely the non-negative definiteness of $\mathrm{J}^{n}[y \mid a, b]$ on the respective class.

\section{Related Criteria of Conjugacy and Disconjugacy}

Suppose that $L_{2 n_{1}}^{1}[u]=0$ and $L_{2 n_{2}}^{2}[u]=0$ are equations (2.3), for which the coefficient functions $p_{j_{1}}^{1}(t),\left(j_{1}=0,1, \ldots, n_{1}\right)$ and $p_{j_{2}}^{2}(t),\left(j_{2}=0,1, \ldots, n_{2}\right)$ satisfy the hypotheses $\left(\mathfrak{S}_{n_{1}}^{+}\right)$and $\left(\mathfrak{S}_{n_{2}}^{+}\right)$. Then for $n$ equal to the greater of $n_{1}$ and $n_{2}$, hypothesis $\left(\mathfrak{Q}_{n}^{+}\right)$is satisfied by the functions $p_{j}=p_{j}^{1}(t)+p_{j}^{2}(t),(j=0,1, \ldots, n)$, with the understanding that for $\alpha=1,2$ we set $p_{j}^{\alpha}(t) \equiv 0$ for $j>n_{\alpha}$. Correspondingly, associated with the equation

$$
L_{2 n}[u] \equiv L_{2 n_{1}}^{1}[u]+L_{2 n_{2}}^{2}[u]=0
$$

one has the quadratic functional 


$$
J^{n}[y \mid a, b]=\int_{a}^{b} \sum_{j=0}^{n}\left[p_{j}^{1}(t)+p_{j}^{2}(t)\right]\left[y^{(j)}(t)\right]^{2} d t \text {, for } y \in \mathscr{D}^{n}[a, b] .
$$

As $\mathscr{D}_{0}^{n}[a, b] \subset \mathscr{D}_{0}^{n_{\alpha}}[a, b]$ for $\alpha=1,2$, as noted at the end of the preceding section we have that if $J^{n_{\alpha}}[y \mid a, b]$ is positive definite on $\mathscr{D}_{0}^{n_{\alpha}}[a, b],(\alpha=1,2)$, then also $\mathbf{J}^{n_{\alpha}}[y \mid a, b]$ is positive definite on $\mathscr{D}_{0}^{n}[a, b]$, and hence in terms of disconjugacy we have the following result.

THEOREM 3.1. If for $\alpha=1,2$ hypothesis $\left(\mathfrak{S}_{n_{\alpha}}^{+}\right)$is satisfied, and each of the equations $L_{2 n_{\alpha}}^{\alpha}[u]=0$ is disconjugate on a subinterval $I_{0}$ of $I$, then $L_{2 n}[u] \equiv$ $L_{2 n_{1}}^{1}[u]+L_{2 n_{2}}^{2}[u]=0$ is also disconjugate on $I_{0}$.

For equations $L_{2 n_{\alpha}}^{\alpha}[u]=0,(\alpha=1,2)$, satisfying hypothesis $\left(\$_{n_{\alpha}}^{+}\right)$, let ${ }^{\alpha} \lambda_{i}^{00}=$ ${ }^{\alpha} \lambda_{j}^{00}[a, b],{ }^{\alpha} u_{j}^{00}(t)={ }^{\alpha} u_{j}^{00}(t \mid a, b)$ denote a set of eigenvalues and eigenfunctions as in Theorem 2.2, while $\lambda_{j}^{00}=\lambda_{j}^{00}[a, b], u_{j}^{00}(t)=u_{j}^{00}(t \mid a, b)$ denotes a similar set for $L_{2 n}[u]=0$. If $n^{-}\left\{J^{n_{1}} / \mathscr{D}_{0}^{n_{1}}[a, b]\right\}=h$, so that ${ }^{1} \lambda_{1}^{\infty} \leq \cdots \leq{ }^{1} \lambda_{h}^{\infty}<0 \leq{ }^{1} \lambda_{h+1}^{\infty}$, then for $i \geq 1$ there exist real constants $c_{1}, \ldots, c_{h+i}$ such that $c_{1}^{2}+\cdots+c_{h+i}^{2}=1$ and $y=\Sigma_{j=1}^{h+i} c_{j} u_{j}^{00}(t)$ satisfies the $h+i-1$ conditions $\int_{a}^{b}{ }^{1} u_{j}^{00}(t) y(t) d t=0,(j=1, \ldots, h)$, and $\int_{a}^{b}{ }^{2} u_{\alpha}^{00}(t) y(t) d t=0,(\alpha=1, \ldots, i-1)$, so that $\int_{a}^{b} y^{2}(t) d t=1$ and

$$
\lambda_{h+i}^{00}[a, b] \geq \mathbf{J}^{n}[y \mid a, b]=\mathbf{J}^{n_{1}}[y \mid a, b]+\mathbf{J}^{n_{2}}[y \mid a, b] \geq \mathbf{J}^{n_{2}}[y \mid a, b] \geq{ }^{2} \lambda_{i}^{00}[a, b] .
$$

In particular, we have the following result extending the conclusion of Theorem 3.1.

THEOREM 3.2. If $s \in I$ and $n^{-}\left\{J^{n_{1}} / \mathscr{D}_{0}^{n_{1}}[s, b]\right\} \leq h$ for all $b \in I_{s}^{+}$, then if for an integer $k>h$ the conjugate point $t_{k}^{+}(s)=t_{k}^{+}\left(s \mid J^{n}\right)=t_{k}^{+}\left(s \mid J^{n_{1}}+J^{n_{2}}\right)$ exists, then ${ }^{2} t_{k-h}^{+}(s)=t_{k-h}^{+}\left(s \mid J^{n}\right)$ exists and ${ }^{2} t_{k-h}^{+}(s) \leq t_{k}^{+}(s)$.

Indeed, under the hypothesis of he theorem one may choose $b=t_{k}^{+}\left(s \mid J^{n}\right)$ so that $\lambda_{k}^{00}[s, b]=0$, and from (3.3) it follows that ${ }^{2} \lambda_{k-h}^{00} \leq 0$, so that the $(k-h)$-th right-hand conjugate point ${ }^{2} t_{k-h}^{+}(s)$ of $s$ relative to $L_{2 n_{2}}^{2}[u]=0$ does not exceed $b=t_{k}^{+}\left(s \mid \mathbf{J}^{n}\right)$.

Of particular interest is the generation of differential equations of higher order through starting with an equation $L_{2 n}[u]=0$ satisfying $\left(\mathfrak{S}_{n}^{+}\right)$with associated functional $\mathrm{J}^{n}[y \mid a, b]$, and deriving an equation $L_{2(n+q)}[u]=0$ satisfying $\left(\mathfrak{Q}_{n+q}^{+}\right)$with corresponding functional $\mathbf{J}^{n+q}[y \mid a, b]$ through the substitution

$$
\mathbf{J}^{n+q}[y \mid a, b]=\mathbf{J}^{n}\left[y^{(a)} \mid a, b\right] \text { for } y \in \mathscr{D}^{n+q}[a, b] \text {. }
$$

THEOREM 3.3. Suppose that $L_{2 n}[u]=0$ satisfies $\left(\mathfrak{S}_{n}^{+}\right)$, and that $L_{2(n+q)}[u]=0$ is the equation associated with the functional $\mathrm{J}^{n+q}[y \mid a, b]$ of (3.4) for $q \geq 1$. If $s \in I$ and $t_{k}^{+}\left(s \mid \mathbf{J}^{n+q}\right)$ exists, then $t_{k}^{+}\left(s \mid \mathbf{J}^{n}\right)$ exists and

$$
t_{k}^{+}\left(s \mid \mathbf{J}^{n}\right) \leq t_{k}^{+}\left(s \mid \mathbf{J}^{n+q}\right)
$$

in particular, for $s_{1} \in I_{s}^{+}$we have $n^{-}\left\{\mathbf{J}^{n} \mid \mathscr{D}_{0}^{n}\left[s, s_{1}\right]\right\} \geq n^{-}\left\{\mathbf{J}^{n+q} / \mathscr{D}_{0}^{n+q}\left[s, s_{1}\right]\right\}$. If $k>$ $q \geq 1$ and $t_{k}^{+}\left(s^{\prime} \mid J^{n}\right)$ exists, then $t_{k-q}^{+}\left(s \mid J^{n+q}\right)$ exists and

$$
t_{k-q}^{+}\left(s \mid J^{n+q}\right) \leq t_{k}^{+}\left(s \mid J^{n}\right) .
$$


For $b=t_{k}^{+}\left(s \mid \mathbf{J}^{n+q}\right)$ let $\lambda_{j}^{00}, u_{j}^{00}(t)$ be a sequence of eigenvalues and eigenfunctions of $B_{00}\left[s, b \mid L_{2 n}\right]$ satisfying the conditions of Theorem 2.2 , and $\hat{\lambda}_{j}^{\circ 0}, \hat{u}_{j}^{\infty 0}(t)$ a corresponding sequence for $B_{00}\left[s, b \mid L_{2(n+q)}\right]$. Then $\hat{\lambda}_{k}^{00}[s, b]=0$ and for $y(t)=$ $\sum_{j=1}^{k} c_{j} \hat{u}_{j}^{00}(t)$ we have $y \in \mathscr{D}_{0}^{n+q}[s, b]$ and $y^{(q)} \in \mathscr{D}_{0}^{n}[s, b]$. Then there exist real constants $c_{1}, \ldots, c_{k}$ such that $c_{1}^{2}+\cdots+c_{k}^{2}=1$ and $\int_{s}^{b} u_{j}^{00}(t) y^{(q)}(t) d t=0,(j=$ $1, \ldots, k-1)$. As $\int_{s}^{b} y^{2}(t) d t=1$, we have also $\int_{s}^{b}\left[y^{(q)}(t)\right]^{2} d t>0$, and the relations

$$
\lambda_{k}^{00}[s, b] \int_{s}^{b}\left[y^{(q)}(t)\right]^{2} d t \leq \mathbf{J}^{n}\left[y^{(q)} \mid s, b\right]=\mathbf{J}^{n+q}[y \mid s, b] \leq \hat{\lambda}_{k}^{00}[s, b]=0
$$

imply that $\lambda_{k}^{00}[s, b] \leq 0$, and consequently that $t_{k}^{+}\left(s \mid \mathbf{J}^{n}\right)$ exists and does not exceed $t_{k}^{+}\left(s \mid \mathbf{J}^{n+q}\right)$. In turn, since $n^{-}\left\{\mathbf{J}^{n} / \mathscr{D}_{0}^{n}\left[s, s_{1}\right]\right\}$ and $n^{-}\left\{\mathbf{J}^{n+q} / \mathscr{D}_{0}^{n+q}\left[s, s_{1}\right]\right\}$ are equal to the number of points on $\left(s, s_{1}\right)$ conjugate to $t=s$ with respect to $L_{2 n}[u]=0$ and $L_{2(n+q)}[u]=0$, respectively, for $s_{1} \in I_{s}^{+}$we have $n^{-}\left\{\mathbf{J}^{n} / \mathscr{D}_{0}^{n}\left[s, s_{1}\right]\right\} \geq$ $n^{-}\left\{\mathbf{J}^{n+q} / \mathscr{D}_{0}^{n+q}\left[s, s_{1}\right]\right\}$.

Now suppose that $k>q \geq 1$ and $t_{k}^{+}\left[s \mid J^{n}\right]$ exists. For $b=t_{k}^{+}\left[s \mid J^{n}\right]$ we then have $\lambda_{k}^{00}[s, b]=0$, and for $y_{1}(t)=\Sigma_{j=1}^{k} c_{j} u_{j}^{00}(t)$ there exist real constants $c_{1}, \ldots, c_{k}$ such that $y(t)=\int_{b}^{t} d s_{q} \int_{b}^{s_{a}} \ldots \int_{b}^{s_{1}} y_{1}(s) d s$ satisfies $\int_{s}^{b} y^{2}(t) d t=1, y(s)=y^{\prime}(s)=\cdots=$ $y^{(q-1)}(s)=0$, and $\int_{s}^{b} \hat{u}_{\alpha}^{00}(t) y(t) d t=0,(\alpha=1, \ldots, k-q-1)$. Then

$$
\hat{\lambda}_{k-q}[s, b] \leq \mathbf{J}^{n+q}[y \mid s, b]=\mathbf{J}^{n}\left[y_{1} \mid s, b\right] \leq \lambda_{k}^{00}[s, b] \int_{s}^{b} y_{1}^{2}(t) d t=0,
$$

and consequently $t_{k-q}^{+}\left(s \mid \mathbf{J}^{n+q}\right)$ exists and does not exceed $b=t_{k}^{+}\left(s \mid \mathbf{J}^{n}\right)$.

For right-hand focal points one has the following results corresponding to the above Theorems 3.2, 3.3 for conjugate points.

THEOREM 3.4. If $s \in I$ and $n^{-}\left\{J^{n_{1}} / \mathscr{D}_{* 0}^{n_{1}}[s, b]\right\} \leq h$ for all $b \in I_{s}^{+}$, then if for an integer $k>h$ the right-hand focal point $\tau_{k}^{+}(s)=\tau_{k}^{+}\left(s \mid J^{n}\right)=\tau_{k}^{+}\left(s \mid J^{n_{1}}+J^{n_{2}}\right)$ exists, then ${ }^{2} \tau_{k-h}^{+}(s)=\tau_{k-h}^{+}\left(s \mid J^{n_{2}}\right)$ exists and ${ }^{2} \tau_{k-h}^{+}(s) \leq \tau_{k}^{+}(s)$.

THEOREM 3.5. If $L_{2 n}[u]=0$ satisfies $\left(\mathfrak{Q}_{n}^{+}\right)$, then for $k=1,2, \ldots$ the right-hand focal point $\tau_{k}^{+}\left(s \mid \mathbf{J}^{n}\right)$ exists iff for $L_{2(n+q)}[u]=0$ the right-hand focal point $\tau_{k}^{+}\left(s \mid \mathbf{J}^{n+q}\right)$ exists and $\tau_{k}^{+}\left(s \mid \mathbf{J}^{n}\right)=\tau_{k}^{+}\left(s \mid \mathbf{J}^{n+q}\right)$.

If $\tau_{k}^{+}\left(s \mid \mathbf{J}^{n+q}\right)$ exists, and for $b=\tau_{k}^{+}\left(s \mid \mathbf{J}^{n+q}\right)$ in the above argument leading to (3.5) the elements of the boundary problems $B_{00}\left[s, b \mid L_{2 n}\right]$ and $B_{00}\left[s, b \mid L_{2(n+q)}\right]$ are replaced by the corresponding elements of $B_{* 0}\left[s, b \mid L_{2 n}\right]$ and $B_{* 0}\left[s, b \mid L_{2(n+q)}\right]$, respectively, then one is led to the conclusion that $\lambda_{k}^{* 0}[s, b] \leq 0$, so that $\tau_{k}^{+}\left(s \mid J^{n}\right)$ exists and does not exceed $b=\tau_{k}^{+}\left(s \mid J^{n+q}\right)$. Moreover, if $\tau_{k}^{+}\left(s \mid J^{n}\right)$ exists and for $b=\tau_{k}^{+}\left(s \mid J^{n}\right)$ one proceeds as in the proof of the second portion of Theorem 3.4 with the elements of $B_{00}\left[s, b \mid L_{2 n}\right]$ and $B_{00}\left[s, b \mid L_{2(n+q)}\right]$ replaced by the respective elements of $B_{* 0}\left[s, b \mid L_{2 n}\right]$ and $B_{* 0}\left[s, b \mid L_{2(n+q)}\right]$, then one obtains the conclusion that $\tau_{k}^{+}\left(s \mid J^{n+a}\right) \leq \tau_{k}^{+}\left(s \mid J^{n}\right)$, since for $y_{1}(t)=\sum_{j=1}^{k} c_{j} u_{j}^{* 0}(t)$ the function $y(t)=$ $\int_{b}^{t} d s_{q} \int_{b}^{s_{a}} \ldots \int_{b}^{s_{1}} y_{1}(s) d s$ defines an element of $\mathscr{D}_{* 0}^{n+q}[a, b]$ without the adjunction of restraints at $t=a$. Consequently, we have $\tau_{k}^{+}\left(s \mid J^{n}\right)=\tau_{k}^{+}\left(s \mid J^{n+q}\right)$ for $k=$ $1,2, \ldots$

Indeed, the result of Theorem 3.5 may be established directly without the 
above comparison theorems, since if $L_{2 n}[u]=0$ is the equation (2.3) for $\mathrm{J}^{n}[y \mid a, b]$ then for $\mathbf{J}^{n+q}[y \mid a, b]=\mathbf{J}^{n}\left[y^{(q)} \mid a, b\right]$ the corresponding equation is $L_{2(n+q)}[u]=(-1)^{q}\left(L_{2 n}\left[u^{(q)}\right]\right)^{(q)}=0$, and in terms of the functions $v_{j}(t \mid u)$ for $L_{2 n}[u]$ as specified by (2.4) the corresponding functions $\hat{v}_{i}(t)=\hat{v}_{j}(t \mid u),(j=1, \ldots, n+$ $q)$, for $L_{2(n+q)}[u]$ are

$$
\begin{aligned}
& \hat{v}_{n+q-i}(t)=v_{n-j}\left(t \mid u^{(q)}\right), \quad(j=0,1, \ldots, n-1), \\
& \hat{v}_{n+q-i}(t)=(-1)^{n-i}\left(L_{2 n}\left[u^{(q)}\right]\right)^{(i-n)}, \quad(i=n, n+1, \ldots, n+q-1) .
\end{aligned}
$$

Consequently, if $u_{1}$ is a solution of $L_{2 n}\left[u_{1}\right]=0$ with $u_{1}^{(k-1)}(b)=0, v_{k}\left(s \mid u_{1}\right)=0$, $(k=1,2, \ldots, n)$, then $u(t)=\int_{b}^{t} d s_{q} \int_{b}^{s_{q}} \ldots \int_{b}^{s_{1}} u_{1}(s) d s$ is a solution of $L_{2(n+q)}[u]=0$ satisfying $\hat{v}_{j}(s \mid u)=0, u^{(j-1)}(b)=0,(j=1, \ldots, n+q)$.

Now for a given $L_{2 n}[u]=0$ satisfying $\left(\mathfrak{B}_{n}^{+}\right)$consider the case wherein there exists an integer $r,(1 \leq r<n)$, and a function $g: I \rightarrow R$ such that $g(t)>0$ for $t \in I$, while $g$ and $1 / g$ belong to $\mathfrak{R}^{\infty}[a, b]$ for arbitrary compact $[a, b] \subset I$. Then we may write

$$
\mathbf{J}^{n}[y \mid a, b]=\hat{\mathbf{J}}^{n}[y \mid a, b]+\mathbf{J}^{r}[y \mid a, b], \text { for } y \in \mathscr{D}_{0}^{n}[a, b],
$$

where

$$
\begin{gathered}
\hat{\mathbf{J}}^{n}[y \mid a, b]=\mathbf{J}^{n-r}\left[y^{(r)} \mid a, b\right], \\
\mathbf{J}^{n-r}[y \mid a, b]=\int_{a}^{b}\left\{\sum_{\gamma=1}^{n-r} p_{r+\gamma}(t)\left[y^{(\gamma)}(t)\right]^{2}+\left[p_{r}(t)-g(t)\right] y^{2}(t)\right\} d t, \\
\mathbf{J}^{r}[y \mid a, b]=\int_{a}^{b}\left\{g(t)\left[y^{(r)}(t)\right]^{2}+\sum_{\beta=0}^{r-1} p_{\beta}(t)\left[y^{(\beta)}(t)\right]^{2}\right\} d t .
\end{gathered}
$$

In view of Theorem 3.2, and the first part of Theorem 3.3, we then have the following result.

THEOREM 3.6. Suppose that the functionals $\mathbf{J}^{n}, \hat{\mathbf{J}}^{n}, \mathbf{J}^{n-r}$ and $\mathbf{J}^{r}$ are defined by (3.7)-(3.10), where hypothesis $\left(\mathfrak{W}_{n}^{+}\right)$is satisfied and $g: I \rightarrow R$ is such that $g(t)>0$, while $g$ and $1 / g$ belong to $\mathfrak{Q}^{\infty}[a, b]$ for arbitrary compact $[a, b] \subset I$. If $s \in I$, and

$$
n^{-}\left\{\mathbf{J}^{n-r} / \mathscr{D}_{0}^{n-r}[s, b]\right\} \leq h \text { for all } b \in I_{s}^{+},
$$

then if for an integer $k>h$ the conjugate point $t_{k}^{+}\left(s \mid J^{n}\right)$ exists the conjugate point $t_{k-h}^{+}\left(s \mid J^{r}\right)$ also exists and $t_{k-h}^{+}\left(s \mid J^{r}\right) \leq t_{k}^{+}\left(s \mid J^{n}\right)$. In particular, if $I$ is of the form $[c, \infty)$, relation (3.11) holds for all $s \in I$ and $b \in I_{s}^{+}$, while the equation

$$
L_{2 r}[u](t) \equiv p_{0}(t) u(t)-\left\{p_{1}(t) u^{\prime}(t)-\left\{\cdots-\left\{g(t) u^{(r)}(t)\right\}^{\prime} \cdots\right\}^{\prime}\right\}^{\prime}=0
$$

is disconjugate on a subinterval $[c, \infty)$ of $I$, then $L_{2 r}[u](t)=0$ is also disconjugate on $\left[c_{0}, \infty\right)$.

In particular, for $n=2$ and $h=0$ the result of Theorem 3.6 generalizes the result of [2, Theorem 5.3] for a particular type of fourth order equation.

For focal points one has under the initial hypothesis of Theorem 3.6 that if $s \in I$ and

$$
n^{-}\left\{\mathbf{J}^{n-r} / \mathscr{D}_{*}^{n-\prime}[s, b]\right\} \leq h \text { for all } b \in I_{s}^{+},
$$


then if for an integer $k>h$ the right-hand focal point $\tau_{k}^{+}\left(s \mid J^{n}\right)$ of $s$ with respect to $L_{2 n}[u]=0$ exists the right-hand focal point $\tau_{k-h}^{+}\left(s \mid J^{r}\right)$ of $s$ with respect to $L_{2 r}[u]=0$ also exists and $\tau_{k-h}^{+}\left(s \mid \mathbf{J}^{r}\right) \leq \tau_{k}^{+}\left(s \mid \mathbf{J}^{n}\right)$.

For a related result involving a given equation $L_{2 n}[u]=0$ satisfying $\left(\$_{n}^{+}\right)$, consider the case wherein there exists an integer $r,(1 \leq r<n)$, and a function $f: I \rightarrow R$ belonging to $\mathfrak{Q}^{\infty}[a, b]$ for arbitrary $[a, b] \subset I$, such that the functional

$$
\check{\mathbf{J}}^{r}[y \mid a, b]=\int_{a}^{b}\left\{f(t)\left[y^{(r)}(t)\right]^{2}-\sum_{\beta=0}^{r-1} p_{\beta}(t)\left[y^{(\beta)}(t)\right]^{2}\right\} d t
$$

satisfies with a given integer $l$ the condition

$$
n^{-}\left\{\check{\mathbf{J}}^{\prime} / \mathscr{D}_{0}^{r}[a, b]\right\} \leq l \text {, for arbitrary }[a, b] \subseteq I \text {. }
$$

In particular, if $p_{\beta}(t) \leq 0$ for $\beta=0,1, \ldots, r-1$ and $t \in I$, then this condition with $l=0$ is satisfied by $f(t) \equiv 0$. Also, if $I$ is a compact subinterval of $R$, then with the aid of elementary inequalities of the general type as those occurring in the proof of Lemma 11.1 of [4, pp. 383-384] one may establish that (3.14) holds with $l=0$ whenever $f(t)$ is equal to a sufficiently large positive constant. Associated with (3.13), now set

so that

$$
\begin{gathered}
\check{\mathbf{J}}^{n-r}[y \mid a, b]=\int_{a}^{b}\left\{\sum_{i=1}^{n-r} p_{r+i}(t)\left[y^{(i)}(t)\right]^{2}+\left[p_{r}(t)+f(t)\right] y^{2}(t)\right\} d t, \\
\check{\mathbf{J}}^{n}[y \mid a, b]=\check{\mathbf{J}}^{n-r}\left[y^{(r)} \mid a, b\right],
\end{gathered}
$$

$$
\mathbf{J}^{n}[y \mid a, b]=\check{\mathbf{J}}^{n}[y \mid a, b]-\check{\mathbf{J}}^{r}[y \mid a, b] \text {, for } y \in \mathscr{D}_{0}^{n}[a, b] .
$$

Then one has the following oscillation theorem.

THEOREM 3.7. Suppose that the functionals $\breve{\mathbf{J}}^{r}, \check{\mathbf{J}}^{n-r}, \check{\mathbf{J}}^{n}$ and $\mathbf{J}^{n}$ are defined by (3.13), (3.15), (3.16), (3.17), where hypothesis $\left(\mathfrak{W}_{n}^{+}\right)$is satisfied and $f: I \rightarrow R$ is such that (3.14) holds. If $s \in I$, and for an integer $k$ the conjugate point $t_{r+1+k}^{+}\left(s \mid \breve{J}^{n-r}\right)$ exists then the conjugate point $t_{k}^{+}\left(s \mid \mathbf{J}^{n}\right)$ exists and $t_{k}^{+}\left(s \mid J^{n}\right) \leq t_{r+l+k}^{+}\left(s \mid \breve{J}^{n-r}\right)$.

Let $b \in I_{s}^{+}$be such that $b=t_{r+l+k}^{+}\left(s \mid \breve{J}^{n-r}\right)$, and denote by $\varphi_{\alpha}(t),(\alpha=1, \ldots, l)$, functions of class $\mathscr{L}[s, b]$ such that $\breve{J}^{r}[y \| s, b] \geq 0$ if $y \in \mathscr{D}_{0}^{\prime}[s, b]$ and $\int_{s}^{b} \varphi_{\alpha}(t) y(t) d t=0,(\alpha=1, \ldots, l)$. Also, let $\check{\lambda}_{j}^{\infty 0}[s, b], \check{u}_{j}^{\infty 0}(t)=\check{u}_{j}^{\infty}(t \mid s, b)$ denote a set of eigenvalues and eigenfunctions of the boundary problem $B_{00}\left[s, b \mid L_{2(n-r)}\right]$ satisfying the conditions of Theorem 2.2 and, as usual, let $\lambda_{j}^{00}[s, b], u_{j}^{00}(t)=$ $u_{j}^{00}(t \mid s, b)$ be a corresponding set of eigenvalues and eigenfunctions of $B_{00}\left[s, b \mid L_{2 n}\right]$. Under the hypotheses of the theorem we then have $\lambda_{r+l+k}^{\infty}[s, b]=0$, and there exists real constants $c_{1}, \ldots, c_{r+l+k}$ for which $y_{1}(t)=\sum_{j=1}^{r+l+k} c_{j} \check{u}_{j}^{00}(t)$ is such that $\int_{s}^{b} y_{1}^{2}(t) d t=1$, and $y(t)=\int_{b}^{t} d s_{r} \int_{b}^{s_{r}} \ldots \int_{b}^{s_{t}} y_{1}(s) d s$ satisfies the $r+l+k-$ 1 conditions

$$
\begin{aligned}
& \text { (i) } y^{(\beta)}(s)=0, \quad(\beta=0, \ldots, r-1), \\
& \text { (ii) } \int_{s}^{b} u_{j}^{00}(t) y(t) d t=0, \quad(j=1, \ldots, k-1), \\
& \text { (iii) } \int_{s}^{b} \varphi_{\alpha}(t) y(t) d t=0, \quad(\alpha=1, \ldots, l) .
\end{aligned}
$$


Then $\int_{s}^{b} y^{2}(t) d t>0$ and $\lambda_{k}[s, b] \int_{s}^{b} y^{2}(t) d t \leq \mathbf{J}^{n}[y \mid s, b]=\check{\mathbf{J}}^{n}[y \mid s, b]-\check{\mathbf{J}}^{\prime}[y \mid s, b] \leq$ $\breve{\mathbf{J}}^{n-r}\left[y_{1} \mid s, b\right] \leq \bar{\lambda}_{r+l+k}^{00}[s, b]=0$, so that $\lambda_{k}[s, b] \leq 0$, and consequently $t_{k}^{+}\left(s \mid \mathbf{J}^{n}\right)$ exists and does not exceed $b=t_{r+l+k}^{+}\left(s \mid \check{\mathbf{J}}^{n-r}\right)$.

As a particular instance one has the following result, which for $n=2, r=1$, generalizes the result of conclusion (b) of [1, Theorem 4.2].

COROLlaRY. Suppose that hypothesis $\left(\mathfrak{S}_{n}^{+}\right)$is satisfied and there exists an integer $r,(1 \leq r<n)$, such that $p_{\beta}(t) \leq 0$ a.e. on $I$ for $\beta=0,1, \ldots, r-1$. If $s \in I$ and relative to the equation

$$
\check{L}_{2(n-r)}[u](t) \equiv p_{r}(t) u(t)-\left\{p_{r+1}(t) u^{\prime}(t)-\left\{\ldots\left\{p_{n}(t) u^{(n-r)}(t)\right\}^{\prime} \ldots\right\}^{\prime}\right\}^{\prime}=0
$$

the $(r+k)$-th conjugate point $\check{t}_{r+k}^{+}\left(s \mid \breve{\mathbf{J}}^{n-r}\right)$ exists, then relative to the equation $L_{2 n}[u]=0$ the $k$-th conjugate point $t_{s}^{+}\left(s \mid \mathbf{J}^{n}\right)$ exists and $t_{k}^{+}\left(s \mid \mathbf{J}^{n}\right) \leq \check{t}_{r+k}^{+}\left(s \mid \mathbf{J}^{n-r}\right)$.

Corresponding to Theorem 3.7 one has for focal points the following result, wherein the alteration of indices is for the same reason as occurred in the comparative situations of Theorems 3.3 and 3.5.

THEOREM 3.8. Suppose that the initial hypotheses of Theorem 3.7 hold. If $s \in I$ and for an integer $k$ the right-hand focal point $\tau_{l+k}^{+}\left(s \mid \mathbf{J}^{n-r}\right)$ exists, then the right-hand focal point $\tau_{k}^{+}\left(s \mid \mathbf{J}^{n}\right)$ exists and $\tau_{k}^{+}\left(s \mid \mathbf{J}^{n}\right) \leq \tau_{l+k}^{+}\left(s \mid \breve{\mathbf{J}}^{n-r}\right)$.

\section{Integro-Differential Problems}

As shown in $[3,7]$, for a large class of self-adjoint systems involving integrodifferential equations and two-point boundary conditions one has generalizations of the comparison and oscillation theorems of the classical Sturmian theory, together with the persistence of the extremizing properties of eigenvalues and eigenfunctions. Present attention will be restricted to a rather limited class of such integro-differential problems, for which there will be noted some basic interrelations with the differential problems considered in the preceding sections. Throughout the present section it will be supposed that the coefficient functions $p_{0}(t), \ldots, p_{n}(t)$ satisfy hypothesis $\left(\mathfrak{S}_{n}^{+}\right)$of Section 2 , and that the kernel functions $k_{0}(t, s), \ldots, k_{n-1}(t, s)$ satisfy the following hypothesis.

$\left(\mathfrak{S}_{\mathrm{K}}\right) k_{\beta}(t, s),(\beta=0,1, \ldots, n-1)$, is a real-valued symmetric function on $I \times I$, and belongs to $\mathfrak{L}^{\infty}([a, b] \times[a, b])$ for arbitrary compact subintervals $[a, b]$ of $I$.

Analogous to the functional $\mathrm{J}^{\mathrm{n}}[y, z \mid a, b]$ of (2.1) we now have

$$
\mathbf{I}^{n}[y, z \mid a, b]=\mathbf{J}^{n}[y, z \mid a, b]+\sum_{\beta=0}^{n-1} \int_{a}^{b} \int_{a}^{b} k_{\beta}(t, s) z^{(\beta)}(t) y^{(\beta)}(s) d t d s,
$$

which is also a symmetric form on $\mathscr{D}^{n}[a, b] \times \mathscr{D}^{n}[a, b]$. Corresponding to the earlier usage, $I^{n}[y, y \mid a, b]$ is abbreviated to $I^{n}[y \mid a, b]$. Associated with $I^{n}[y \mid a, b]$ is the integro-differential equation

$$
\Lambda_{2 n}[u \mid a, b](t) \equiv p_{0}(t) u(t)+\int_{a}^{b} k_{0}(t, s) u(s) d s-v_{1}^{\prime}(t),
$$


where, corresponding to (2.4), the functions $v_{i}(t)=v_{i}(t \mid u)=v_{i}(t|u| a, b),(i=$ $1, \ldots, n)$, are defined recursively as

$$
\begin{aligned}
& v_{n}(t)=p_{n}(t) u^{(n)}(t), \\
& v_{n-i}(t)=p_{n-j}(t) u^{(n-j)}(t)+\int_{a}^{b} k_{n-j}(t, s) u^{(n-j)}(s) d s-v_{n-j+1}^{\prime}(t), \\
& (j=1, \ldots, n-1) .
\end{aligned}
$$

Self-adjoint boundary problems involving the integro-differential equation

$$
\Lambda_{2 n}[u \mid a, b](t)-\lambda u(t)=0,
$$

and two-point boundary conditions possess many properties of the self-adjoint differential problems to which they reduce when all the kernel functions $k_{\beta}(t, s)$, $(\beta=0,1, \ldots, n-1)$, are identically zero, although many of the basic techniques that are commonly employed for differential systems are no longer available for the integro-differential problems. In particular, for $(p, q)$ any one of the index sets $(0,0),\left({ }^{*}, 0\right),(0, *)$ the boundary problem $I B_{p q}\left[a, b \mid \Lambda_{2 n}\right]$, involving (4.4) and the corresponding boundary conditions $\Delta_{p q}[a, b]$ of (2.8), possesses properties that are verbatim those of Theorem 2.2 when one replaces $L_{2 n}, \mathrm{~J}^{n}, B_{p q}$ by $\Lambda_{2 n}, \mathbf{I}^{n}$, $I B_{p q}$, respectively. For these results, including those corresponding to conclusions (a)-(d) of Theorem 2.2, see [3; Secs. 4, 5, 6 and 7; Secs. 5, 6]; for a result corresponding to conclusion (e) of Theorem 2.2, see [3, Lemma 6.2]. In this section we shall use these results without further detailed statements. Also, we shall employ the same notations $u_{j}^{p q}(t)=u_{j}^{p q}(t \mid a, b)$ and $\lambda_{j}^{p q}=\lambda_{j}^{p q}[a, b]$ for eigenfunctions and associated eigenvalues of the boundary problems $I B_{p q}$ as used earlier for the corresponding differential boundary problems. Although specified by the employed notation, it is to be emphasized that the end-points $a$ and $b$ of the interval on which the boundary problem $I B_{p q}$ is considered enter specifically in the integral terms of $\Lambda_{2 n}[u \mid a, b]$ and also in the values of the associated functions $v_{j}(t \mid u)=v_{j}(t|u| a, b),(j=1, \ldots, n-1)$. In particular, if $s \in I$ the statement that a value $s_{1} \in I_{s}^{+}$is a conjugate point $t_{j}^{+}\left(s \mid I^{n}\right)$ to $s$ means that there exists a non-identically vanishing solution $u(t)$ of $\Lambda_{2 n}\left[u \mid s, s_{1}\right](t)=0$ satisfying $u^{(\alpha-1)}(s)=0=u^{(\alpha-1)}\left(s_{1}\right),(\alpha=1, \ldots, n)$; correspondingly, $s_{1} \in I_{s}^{+}$is a focal point $\tau_{j}^{+}\left(s \mid I^{n}\right)$ of $s$ if there is a non-identically vanishing solution $u(t)$ of $\Lambda_{2 n}\left[u \mid s, s_{1}\right](t)=0$ satisfying $v_{\alpha}\left(s|u| s, s_{1}\right)=0, u^{(\alpha-1)}\left(s_{1}\right)=0,(\alpha=1, \ldots, n)$.

Now if $y \in \mathscr{D}_{0}^{n}[a, b]$ or $y \in \mathscr{D}_{* 0}^{n}[a, b]$, then for $1 \leq r<n$ and $0 \leq \alpha<r$ we have

$$
y^{(\alpha)}(t)=\int_{t}^{b} \kappa_{\alpha r}(t, s) y^{(r)}(s) d s, \quad t \in[a, b]
$$

with

$$
\begin{aligned}
& \kappa_{\alpha}(t, s)=-\frac{\left[-(s-t)_{+}\right]^{r-\alpha-1}}{(r-\alpha-1) !}, 0 \leq \alpha \leq r-2, \\
& \kappa_{r-1, r}(t, s)=-[\operatorname{sgn}(s-t)]_{+},
\end{aligned}
$$

where, in general, $f_{+}=f$ if $f \geq 0$ and $f_{+}=0$ if $f<0$.

Therefore, if $y$ belongs to either $\mathscr{D}_{0}^{n}[a, b]$ or $\mathscr{D}_{* 0}^{n}[a, b]$, for $\alpha=0,1, \ldots, r-1$, 
we have

$$
\begin{gathered}
\int_{a}^{b} p_{\alpha}(t)\left[y^{(\alpha)}(t)\right]^{2} d t+\int_{a}^{b} \int_{a}^{b} k_{\alpha}(t, s) y^{(\alpha)}(t) y^{(\alpha)}(s) d t d s \\
=\int_{a}^{b} \int_{a}^{b} K_{\alpha r}(t, s) y^{(r)}(t) y^{(r)}(s) d t d s,
\end{gathered}
$$

where

$$
K_{\alpha r}(t, s)=\int_{a}^{b} p_{\alpha}(\tau) \kappa_{\alpha r}(\tau, t) \kappa_{\alpha r}(\tau, s) d \tau+\int_{a}^{b} \int_{a}^{b} k_{\alpha}(\tau, \sigma) \kappa_{\alpha r}(\tau, t) \kappa_{\alpha r}(\sigma, s) d \tau d \sigma .
$$

Consequently, for $K_{r}(t, s)=k_{r}(t, s)+\sum_{\alpha=0}^{r-1} K_{\alpha r}(t, s)$ we have

$$
\mathbf{I}^{n}[y \mid a, b]=\hat{\mathbf{I}}^{n}[y \mid a, b] \text {, for } y \in \mathscr{D}_{0}^{n}[a, b] \text { or } y \in \mathscr{D}_{* 0}^{n}[a, b] \text {, }
$$

where

$$
\hat{\mathbf{I}}^{n}[y \mid a, b]=\mathbf{I}^{n-r}\left[y^{(r)} \mid a, b\right],
$$

with

$$
\begin{aligned}
\mathbf{I}^{n-r}[z \mid a, b]= & \int_{a}^{b}\left\{p_{n}(t)\left[z^{(n-r)}(t)\right]^{2}+\cdots+p_{r}(t) z^{2}(t)\right\} d t \\
& +\sum_{\beta=0}^{n-r-1} \int_{a}^{b} \int_{a}^{b} \tilde{k}_{\beta}(t, s) z^{(\beta)}(t) z^{(\beta)}(s) d t d s,
\end{aligned}
$$

and

$$
\tilde{k}_{0}(t, s)=K_{r}(t, s), \tilde{k}_{i}(t, s)=k_{r+i}(t, s), \quad(i=1, \ldots, n-r-1) .
$$

Associated with (4.8) we have the integro-differential equation

$$
\Lambda_{2(n-r)}[\tilde{u} \mid a, b](t) \equiv p_{r}(t) \tilde{u}(t)+\int_{a}^{b} K_{r}(t, s) \tilde{u}(s) d s-\tilde{v}_{1}^{\prime}(t)=0,
$$

where the $\tilde{v}_{j}(t)=\tilde{v}_{j}(t \mid u)=\tilde{v}_{j}(t|u| a, b),(j=1, \ldots, n-r)$, are defined recursively as

$$
\begin{aligned}
\tilde{v}_{n-r}(t)= & p_{n}(t) \tilde{u}^{(n-r)}(t), \\
\tilde{v}_{n-r-i}(t)= & p_{n-i}(t) \tilde{u}^{(n-r-i)}(t)+\int_{a}^{b} k_{n-i}(t, s) \tilde{u}^{(n-r-i)}(s) d s \\
& -\tilde{v}_{n-r-i+1}^{\prime}(t), \quad(i=1, \ldots, n-r-1) .
\end{aligned}
$$

Moreover, corresponding to the discussion following the proof of Theorem 3.5, we have that the integro-differential equation for $\hat{\mathbf{I}}^{n}[y \mid a, b]=\mathbf{I}^{n-r}\left[y^{(r)} \mid a, b\right]$ is $\hat{\Lambda}_{2 n}[u \mid a, b] \equiv(-1)^{r}\left\{\Lambda_{2(n-r)}\left[u^{(r)} \mid a, b\right]\right\}^{(r)}=0$. Also, in view of $(4.7)$ it follows that for $(p, q)$ equal to $(0,0)$ or $\left({ }^{*}, 0\right)$ a value $\lambda$ is an eigenvalue of $I B_{p q}\left[a, b \mid \Lambda_{2 n}\right]$ of multiplicity $k$ iff this $\lambda$ is an eigenvalue of $I B_{p q}\left[a, b \mid \hat{\Lambda}_{2 n}\right]$ of multiplicity $k$, where $\hat{\Lambda}_{2 n}$ denotes the corresponding (4.2) for $\hat{\mathbf{I}}^{n}[y \mid a, b]=I^{n-r}\left[y^{(r)} \mid a, b\right]$.

Each of the results of Section 3 may be extended to integro-differential equations of the sort introduced above. For brevity, however, only the following analogue of results of Theorems 3.3 and 3.5 will be given specifically. 
THEOREM 4.1. Suppose that hypotheses $\left(\mathfrak{\mathscr { Q }}_{n}^{+}\right)$and $\left(\mathfrak{S}_{K}\right)$ are satisfied, and that $\mathrm{I}^{n}, \mathrm{I}^{n-r}$ are defined as in (4.7), (4.8), (4.9).

(i) If $s \in I$ and $t_{k}^{+}\left(s \mid \mathbf{I}^{n}\right)$ exists, then $t_{k}^{+}\left(s \mid \mathbf{I}^{n-r}\right)$ exists and $t_{k}^{+}\left(s \mid \mathbf{I}^{n-r}\right) \leq t_{k}^{+}\left(s \mid \mathbf{I}^{n}\right)$. If $k>n-r \geq 1$ and $t_{k}^{+}\left(s \mid \mathbf{I}^{n-r}\right)$ exists, then $t_{k-n+r}^{+}\left(s \mid \mathbf{I}^{n}\right)$ exists and $t_{k-n+r}^{+}\left(s \mid \mathbf{I}^{n}\right) \leq$ $t_{k}^{+}\left(s \mid \mathbf{I}^{n-r}\right)$.

(ii) If $s \in I$ then the right-hand focal point $\tau_{k}^{+}\left(s \mid \mathbf{I}^{n-r}\right)$ exists iff $\tau_{k}^{+}\left(s \mid \mathbf{I}^{n}\right)$ exists, and $\tau_{k}^{+}\left(s \mid \mathbf{I}^{n-r}\right)=\tau_{k}^{+}\left(s \mid \mathbf{I}^{n}\right)$.

Inparticular, for $r=n-1$ the functional (4.8) becomes

$$
\begin{aligned}
\mathbf{I}^{1}[z \mid a, b]= & \int_{a}^{b}\left\{p_{n}(t)\left[z^{\prime}(t)\right]^{2}+p_{n-1}(t) z^{2}(t)\right\} d t \\
& +\int_{a}^{b} \int_{a}^{b} K_{n-1}(t, s) z(t) z(s) d t d s .
\end{aligned}
$$

In this instance we also have $\tilde{v}_{1}(t)=p_{n}(t) \tilde{u}^{\prime}(t)$, and (4.10) reduces to the second order integro-differential equation

$$
\Lambda_{2}[\tilde{u} \mid a, b](t) \equiv p_{n-1}(t) \tilde{u}(t)+\int_{a}^{b} K_{n-1}(t, s) \tilde{u}(s) d s-\left[p_{n}(t) \tilde{u}^{\prime}(t)\right]^{\prime}=0 .
$$

\section{REFERENCES}

1 J.H. Barrett. Oscillation theory of ordinary linear differential equations. Advances in Math. 3 (1969), 415-509.

2 W. Leighton and Z. Nehari. On the oscillation of self-adjoint linear differential equations of the fourth order. Trans. Amer. Math. Soc. 89 (1958), 325-377.

3 W.T. Reid. An integro-differential boundary value problem. Amer. J. Math. 60 (1938), 257-292.

4 W.T. Reid. Ordinary Differential Equations (New York: Wiley, 1971).

5 W.T. Reid. A disconjugacy criterion for higher order linear vector differential equations. Pacific J. Math. 39 (1971), 795-806.

6 W.T. Reid. Variational aspects of oscillation phenomena for higher order differential equations. $J$. Math. Anal. Appl. 40 (1972), 446-470.

7 W.T. Reid. Related self-adjoint differential and integro-differential systems. J. Math. Anal. Appl. 54 (1976), 89-114. 